\title{
La maîtrise du temps d'irrigation au sein des oasis alimentées par des aflâj
}

Étude de cas à Adam (Sultanat d'Oman)

The management of irrigation time in the oasis irrigated by a falaj : case study

in Adam (Sultanate of Oman)

\section{Julien Charbonnier}

\section{(2) OpenEdition}

\section{Journals}

Édition électronique

URL : http://journals.openedition.org/ethnoecologie/1471

DOI : 10.4000/ethnoecologie.1471

ISSN : 2267-2419

\section{Éditeur}

Laboratoire Eco-anthropologie et Ethnobiologie

\section{Référence électronique}

Julien Charbonnier, "La maîtrise du temps d'irrigation au sein des oasis alimentées par des aflâj », Revue d'ethnoécologie [En ligne], 4 | 2013, mis en ligne le 31 décembre 2013, consulté le 21 avril 2019 URL : http://journals.openedition.org/ethnoecologie/1471; DOI : 10.4000/ethnoecologie.1471

Ce document a été généré automatiquement le 21 avril 2019

Revue d'ethnoécologie est mis à disposition selon les termes de la licence Creative Commons Attribution - Pas d'Utilisation Commerciale - Pas de Modification 4.0 International. 


\title{
La maîtrise du temps d'irrigation au sein des oasis alimentées par des aflâj
}

\author{
Étude de cas à Adam (Sultanat d'Oman) \\ The management of irrigation time in the oasis irrigated by a falaj : case study \\ in Adam (Sultanate of Oman)
}

Julien Charbonnier

«L'oasis n'est pas une simple juxtaposition de
jardins mais un groupement d'intérêts à la fois
solidaires et concurrents, dont la structure
s'inscrit (...) non seulement dans l'espace, mais
aussi dans le temps. » (Trousset $1986: 165$ )

1 Les oasis omanaises sont alimentées par un système d'acquisition de l'eau connu sous le nom de falaj (pl. aflâj). Ce vocable désigne plusieurs types d'ouvrages : de simples canaux captant l'eau des sources (falaj 'aynî), des digues dérivant les crues des wâdî (falaj ghaylî) ou des canalisations drainantes, équivalentes du qanât iranien (falaj dầûdî̀). Ces dernières sont les plus répandues, notamment sur le piémont des montagnes d'Oman. Un falaj dầûdî se compose d'une galerie souterraine creusée en tunnel, très légèrement inclinée, reliée à la surface par une série d'accès verticaux implantés à intervalle régulier. En amont, la galerie traverse une nappe d'eau qu'elle draine vers l'aval par simple gravité. Sa pente étant toujours plus faible que celle du terrain dans lequel elle est creusée, la galerie drainante finit par rejoindre la surface. Les regards permettent d'évacuer les déblais lors du creusement de la galerie, de la ventiler et, par la suite, de la nettoyer. Dans cet article, nous traiterons uniquement de ce type d'ouvrage. Par soucis de clarté, nous le désignerons par le terme falaj uniquement.

2 On ne peut interrompre le flot qui s'écoule des aflâj, il doit donc être distribué en permanence entre les irrigants. Chacun d'entre eux possède ainsi un certain nombre de parts d'eau, réparties de manière irrégulière ${ }^{1}$ le long d'un cycle de plusieurs jours (appelé dawrân). Le cycle de l'eau se subdivise de différentes manières suivant les oasis. Dans 
nombres d'entre elles, l'unité de base est appelée athar et dure environ 30 minutes $^{2}$. L' athar peut lui-même se fractionner en sous-unités : demis, quarts, etc. Une part d'eau peut être constituée de plusieurs athar contigus.

Ce mode de partage est bien documenté (al-Ghafri et al. 2004 ; Dutton 1989; Nash 2011; Wilkinson 1977). On possède toutefois peu d'informations sur la gestion concrète de l'eau au sein des oasis omanaises et notamment sur la manière dont cette répartition temporelle s'inscrit dans l'espace.

La présente étude a ainsi pour objectif de combler cette lacune. Elle prend pour cadre l'oasis d'Adam, localisée sur le piémont méridional (Figure 1), à environ $60 \mathrm{~km}$ au sud de Nizwâ. Depuis 2007, cette région est l'objet d'une prospection menée par une mission archéologique française. Créée par feu Serge Cleuziou (Université de Paris 1) et successivement dirigée par Jessica Giraud (Ifpo-Irak, antenne d'Erbil), puis Guillaume Gernez (Université de Paris 1). Cette prospection a pour objectif de réaliser la carte archéologique d'Adam et de comprendre l'évolution du peuplement sur les marges du Rub' al-Khâlî, depuis le Néolithique jusqu'à nos jours. L'étude de l'oasis, passée et présente, fait partie intégrante de ce programme. L'ANR Phoenix, dirigé par Margareta Tengberg, a soutenu les missions d'étude portant sur l'oasis d'Adam.

Figure 1. Carte de l'extrémité septentrionale du Sultanat d'Oman

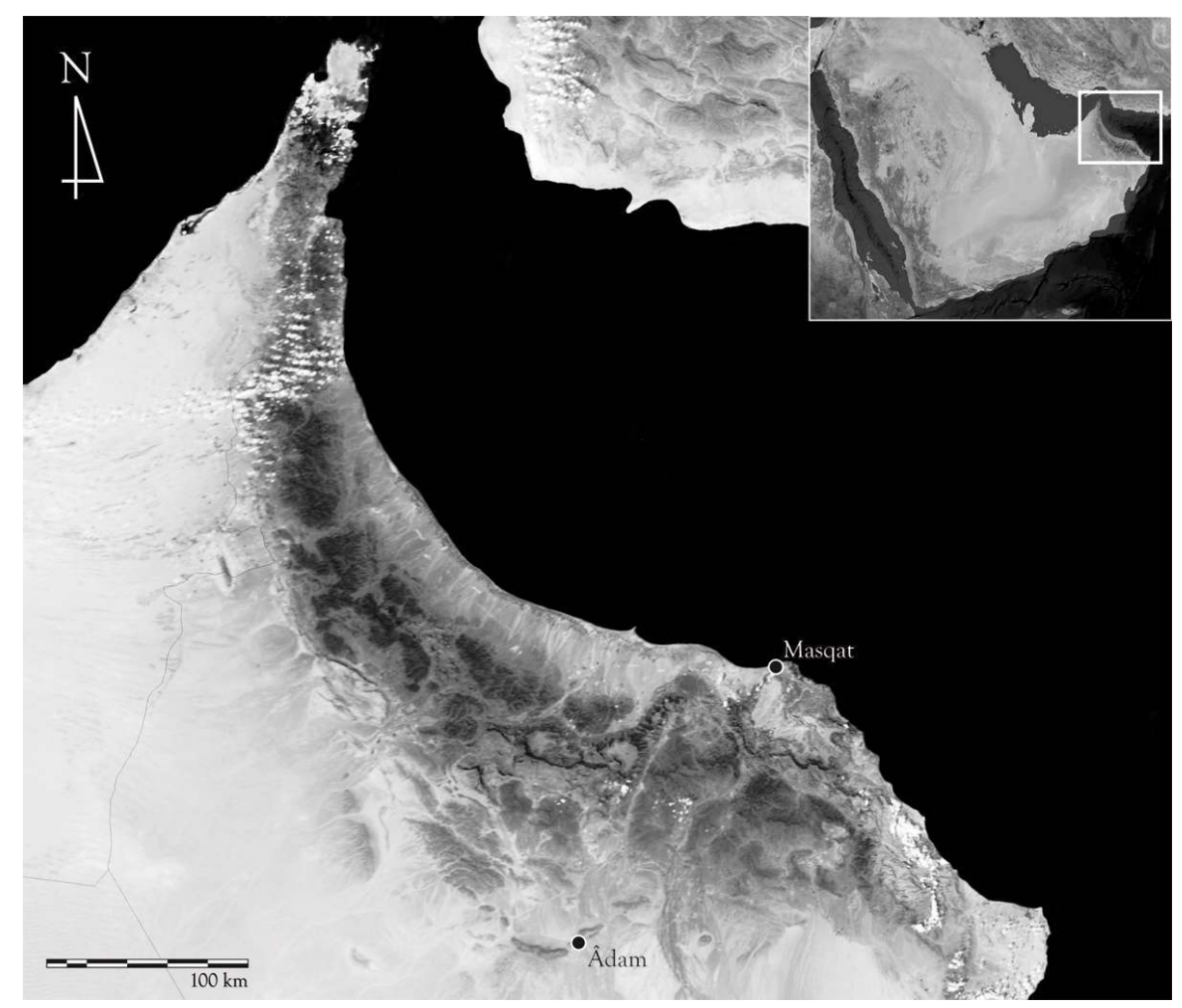

Infographie Julien Charbonnier

5 La cartographie des aflâj et des canaux alimentant la palmeraie et l'enregistrement des aménagements en lien avec la pratique de l'irrigation furent réalisés au cours de trois missions de terrain : janvier 2007, janvier-février 2010 et janvier-février 2011. La dernière campagne fut également en partie consacrée à l'étude des méthodes de partage de l'eau. 
Dans un premier temps, nous introduirons l'oasis et les aflâj d'Adam avant de présenter plus en détail le cadre de notre travail : le falaj al-Mâleh. Seront alors successivement étudiés : le cycle de l'eau de cet ouvrage et l'outil servant à calculer les athar au cours de la journée. Puis nous aborderons la question des modalités spatiales du partage de l'eau dans le système d'irrigation. Celle-ci nous amènera à réfléchir sur les raisons justifiant une telle organisation.

\section{L'oasis et les aflâj d'Adam}

7 Vaste d'environ 100 ha, l'oasis d'Adam est établie entre deux anticlinaux calcaires, le Jabal Muḍmâr à l'est et le Jabal al-Ḥandalî à l'ouest. Comme elle est trop éloignée des montagnes d'al-Ḥajar, les crues du wâdî voisin l'atteignent rarement et ne peuvent donc être utilisées pour l'irrigation. Pour s'implanter, la population locale n'a eu d'autre choix que d'exploiter la nappe d'eau souterraine.

8 Sa palmeraie est alimentée par quatre aflâj qui se déploient dans et autour du lit du wâdî al-Gharbî : falaj al-Mâleh, falaj al-'Ayn, falaj al-Shâr'a et falaj al-Filaij (Figure 2). Le premier s'étire sur environ $4500 \mathrm{~m}$ au pied du Jabal al-Handalî et alimente l'extrémité nord-ouest de l'oasis. Long d'environ $4200 \mathrm{~m}$, le falaj al-'Ayn est quant à lui implanté à l'ouest du Jabal Muḍmâr et irrigue les parcelles situées à l'est et au sud-est de la palmeraie. La partie occidentale de cette dernière est alimentée par le falaj al-Shâr ${ }^{\mathfrak{a}} a$, qui mesure environ $2700 \mathrm{~m}$ de long. Le falaj al-Filaij parcourt environ $3000 \mathrm{~m}$ avant de sortir de terre au sud de l'oasis, où il approvisionne une petite palmeraie indépendante.

Figure 2. Carte du réseau de falaj et de l'oasis d'Adam

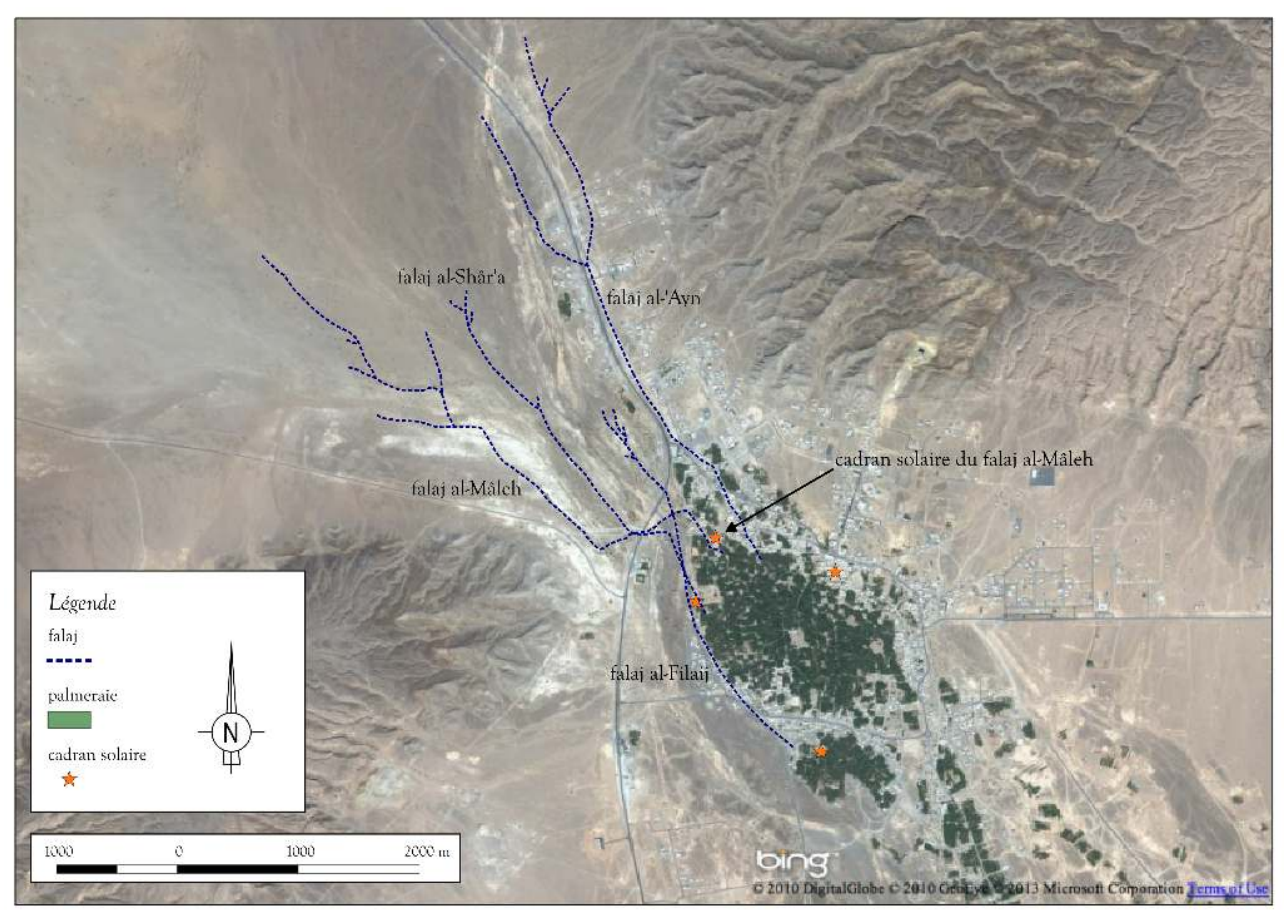

Infographie Julien Charbonnier (c) Mission archéologique française à Adam

Lors de nos premières visites dans l'oasis, il nous a semblé que l'eau n'était pas répartie suivant une logique spatiale, comme cela s'observe dans d'autres régions d'Arabie 
(Yémen). Les parcelles n'étaient pas irriguées les unes à la suite des autres, l'eau pouvant transiter d'une extrémité à l'autre du système d'irrigation entre deux tours. Nous avons donc consacré notre troisième campagne à l'étude précise de ce mode de partage. Notre objectif était d'enregistrer le parcours de l'eau au cours de plusieurs journées : les champs alimentés devant être localisés dans l'oasis à l'aide d'un GPS et leurs propriétaires répertoriés.

Le falaj al-Mâleh a été choisi pour mener à bien ce travail, car il présente l'avantage d'être encore utilisé par un nombre élevé de personnes. Le réseau de canaux qui lui est associé se développe par ailleurs sur une étendue raisonnable - de 600 à $700 \mathrm{~m}$ de long au maximum - si bien qu'il est possible de le parcourir à pied d'amont en aval - afin de localiser les parcelles irriguées -, puis de revenir au lieu où se calculent les parts d'eau en moins de 30 minutes (la durée d'un athar).

\section{Le cycle de l'eau du falaj al-Mâleh}

11 L'eau qui s'écoule du falaj al-Mâleh est répartie entre irrigants sur un cycle de quatorze jours, qui débute un vendredi sur deux. Les journées de vingt-quatre heures sont appelées badda. Chacune d'entre elles est divisée en quarante-huit athar, vingt-quatre diurnes et vingt-quatre nocturnes. Tous les badda sont différents les uns des autres en terme de répartition de l'eau, mais ils se reproduisent à l'identique, ou presque ${ }^{3}$, au cours de chaque cycle.

12 Les badda du falaj al-Mâleh sont les suivants :

$1^{\mathrm{er}}$ vendredi : al-Zayda

$1^{\text {er }}$ samedi : al-Manzarya

$1^{\mathrm{er}}$ dimanche : al-Bula

$1^{\mathrm{er}}$ lundi : al-Khâldiya

$1^{\mathrm{er}}$ mardi : Banî Qâssim

$1^{\mathrm{er}}$ mercredi: Banî Bû 'Alî

$1^{\text {er }}$ jeudi : Banî Salt

$2^{\mathrm{e}}$ vendredi : al-Q'âida

$2^{\mathrm{e}}$ samedi : Banî Ismấîl

$2^{\mathrm{e}}$ dimanche : al-Bâda

$2^{\mathrm{e}}$ lundi : $a$-Rub'a

$2^{\mathrm{e}}$ mardi : Banî Qâssim

$2^{\mathrm{e}}$ mercredi : Banî Bû 'Alî

$2^{\mathrm{e}}$ jeudi : al-Wâdî.

13 Avant l'introduction des montres à quartz et de l'heure universelle, les athar étaient calculés à l'aide des astres : le soleil et les étoiles. Comme la durée du jour varie suivant les saisons, entre 11 et 13 heures à la latitude de l'Oman, les athar de jour sont donc moins longs en hiver qu'en été. Inversement pour ceux de nuit.

14 Au cours de la nuit, la course des étoiles permettait de déterminer le début de chaque athar. Plusieurs techniques ont été documentées par H. Nash $(2009$; 2011). De manière générale, l'observateur se plaçait à un endroit précis - qui pouvait varier suivant les saisons et même au cours de la nuit - et de là attendait le lever de certaines étoiles ou leur passage près de repères situés à l'horizon. Cette méthode n'est plus employée à Adam de 
nos jours. Les athar diurnes y sont en revanche toujours calculés à l'aide de cadrans solaires.

\section{La muḥậara : la maîtrise du temps}

Il existe quatre cadrans solaires à Adam, un par falaj (Figure 2). Celui correspondant au falaj al-Mâleh est implanté au nord de l'oasis, à proximité de l'habitat et à une centaine de mètres du débouché à l'air libre de la galerie souterraine.

Le cadran est enclos par quatre murs bas qui forment un quadrilatère irrégulier mesurant environ $28 \mathrm{~m}$ d'est en ouest et $11 \mathrm{~m}$ du nord au sud (Figures 3 et 4). Le style, la tige verticale dont l'ombre marque les heures, est placé dans la partie méridionale de cet espace, en position centrale. Les irrigants l'appellent khashaba, malgré le fait qu'il soit aujourd'hui en fer ${ }^{4}$. Par extension, ce terme peut également désigner le cadran. Muhâạara est le nom donné au lieu où ce dernier est installé5. Le style mesure $172 \mathrm{~cm}$ de haut pour $2,5 \mathrm{~cm}$ de diamètre. Au nord, il est flanqué par sept lignes de plots métalliques (mismâr), orientées est-ouest. Chaque ligne comporte vingt-deux ou vingt-quatre plots, dont la hauteur varie entre 3 et $9 \mathrm{~cm}$. Situées aux extrémités méridionales et septentrionales, les lignes les plus courtes sont complétées par deux repères virtuels, qui ne sont pas matérialisés au sol, mais qui sont pris en compte par les utilisateurs. Ceux-ci sont situés dans l'axe du style, au centre du cadran.

Figure 3. Le cadran solaire du falaj al-Mâleh, vu depuis l'est

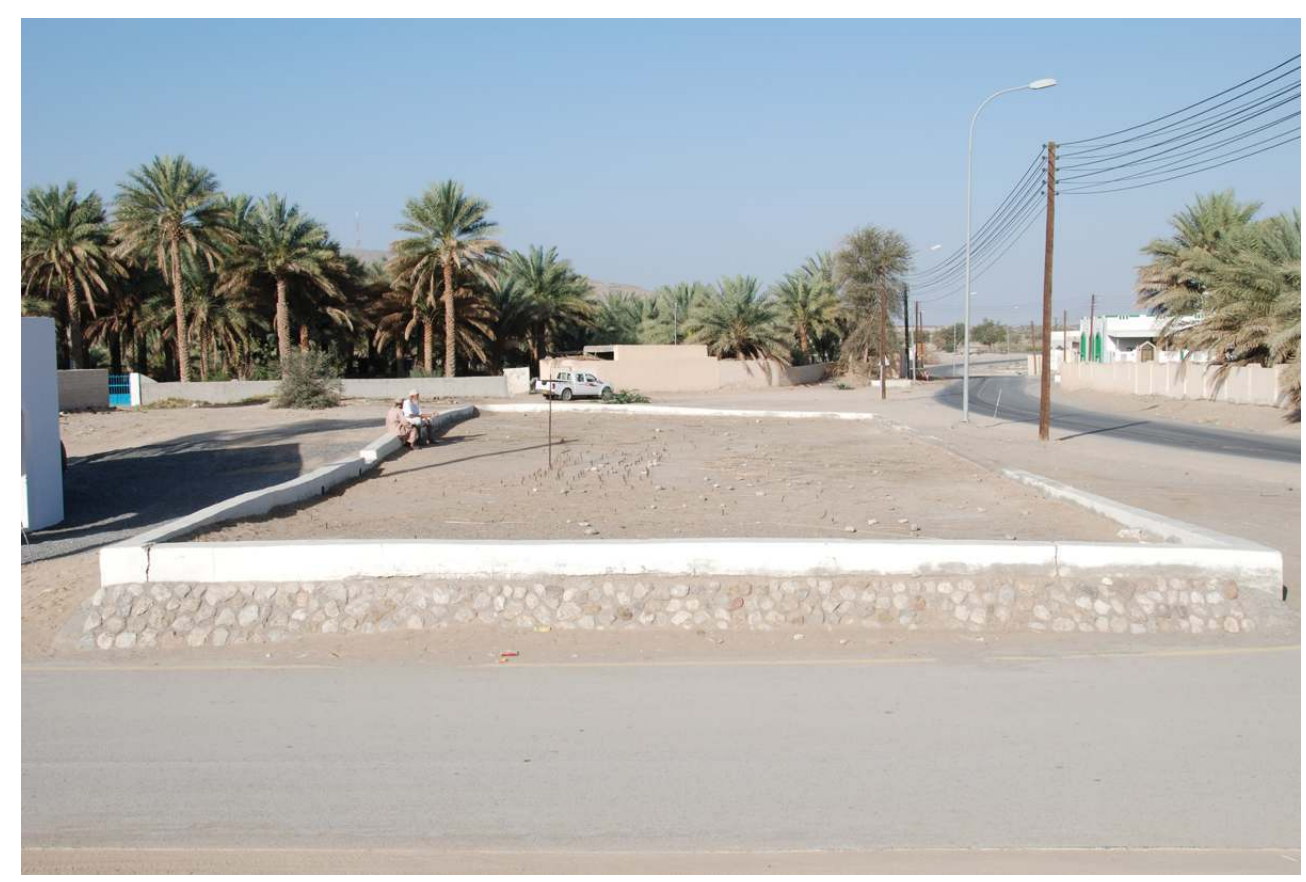

(C) Julien Charbonnier 
Figure 4. Plan du cadran solaire du falaj al-Mâleh

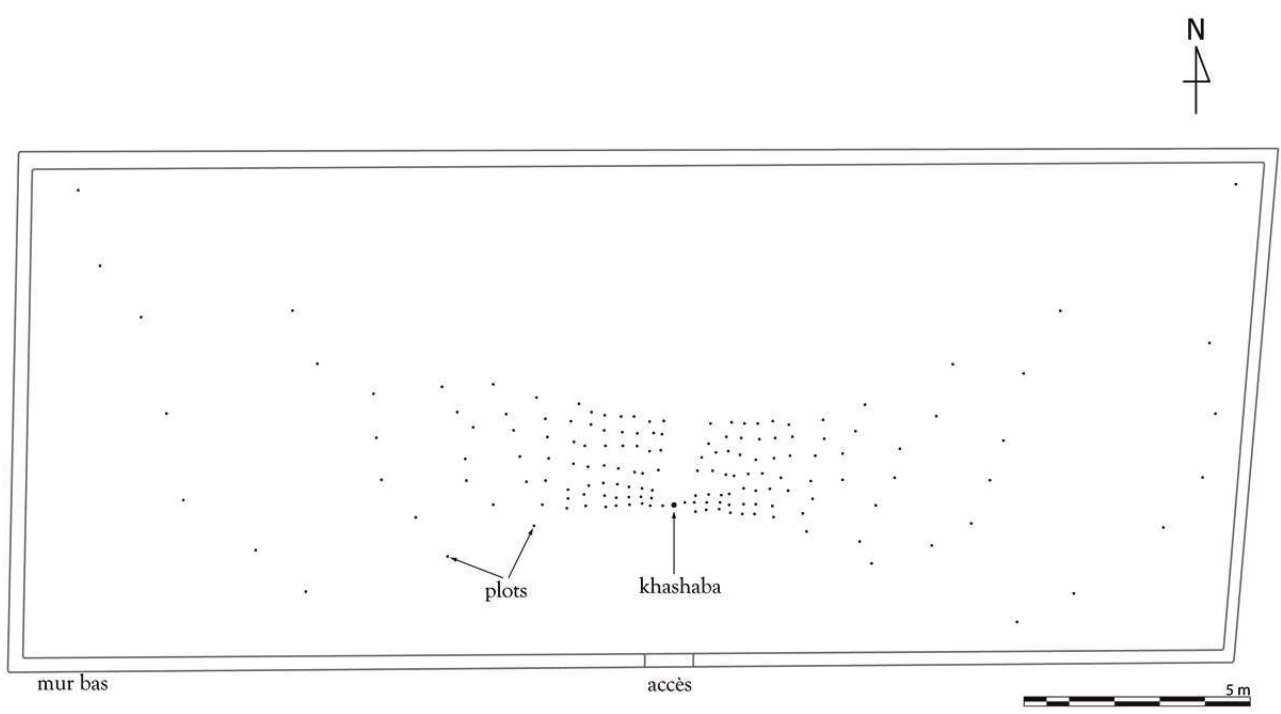

Relevé Julien Charbonnier

17 Au sein de chaque ligne, l'intervalle entre deux plots représente un athar. Au cours de la journée, chaque ayant droit vient placer une petite baguette de bois, appelée khațab, le long du repère correspondant à l'heure de début de son tour d'eau (Figure 5). Il la cale avec des pierres puis il attend que l'extrémité de l'ombre du style la touche pour la retirer et se diriger avec célérité vers le falaj (Figure 6). Il déplace alors une série de vannes mobiles dans des partiteurs afin de dévier l'eau vers la parcelle qu'il souhaite irriguer (Figure 7) ${ }^{6}$. En fin de journée, ou lorsque le temps est nuageux, l'irrigant se place contre la khashaba et tapote du bout du doigt son sommet de manière à mieux distinguer l'extrémité de son ombre. Lorsque les conditions atmosphériques rendent le cadran inutilisable, la montre à quartz est employée et on prend en compte les horaires des jours précédents. 
Figure 5. Un irrigant posant une baguette sur le cadran solaire

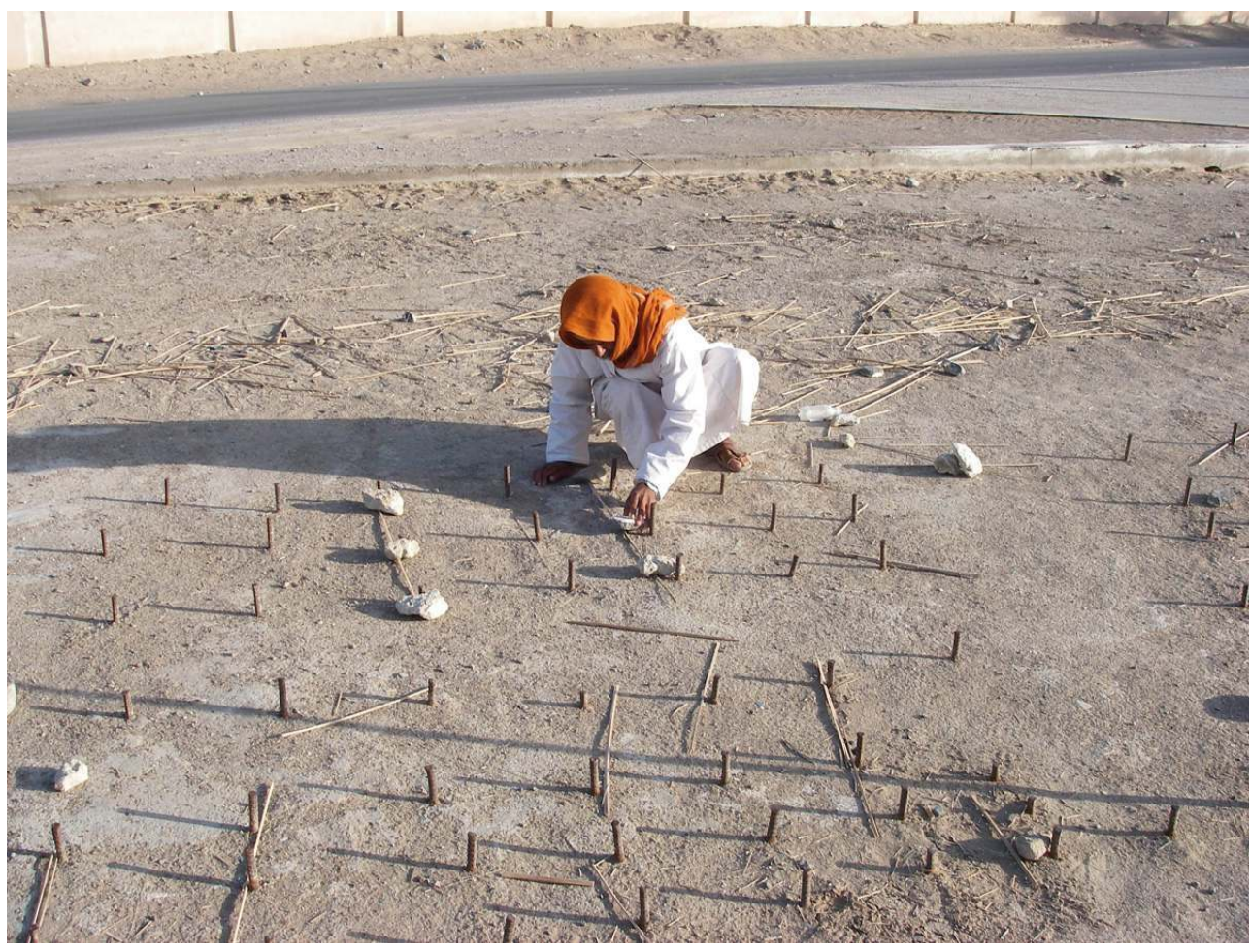

(c) Julien Charbonnier

Figure 6. L'ombre du style se rapproche d'une baguette

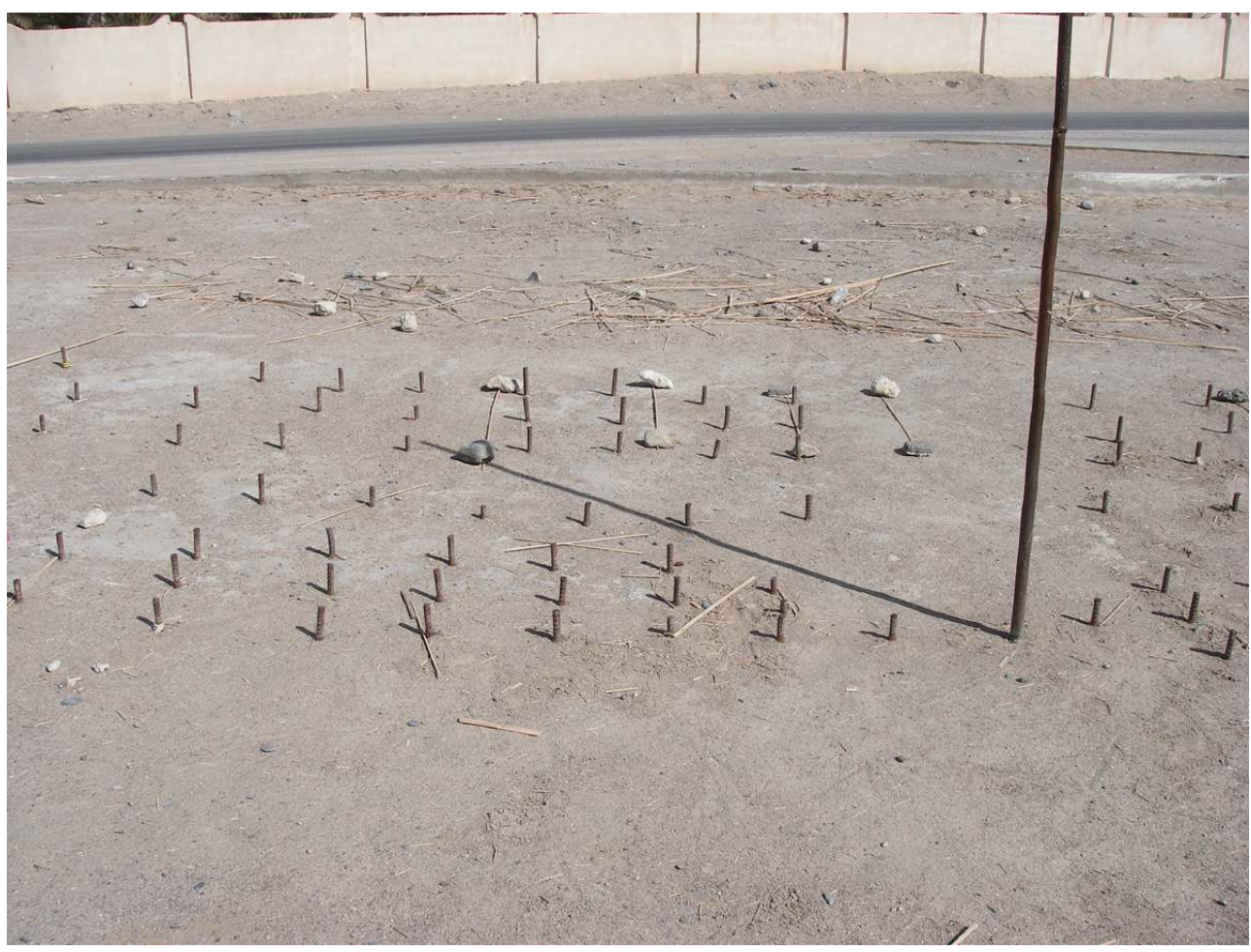

(c) Julien Charbonnier 
Figure 7. Un irrigant déplace une porte de vanne

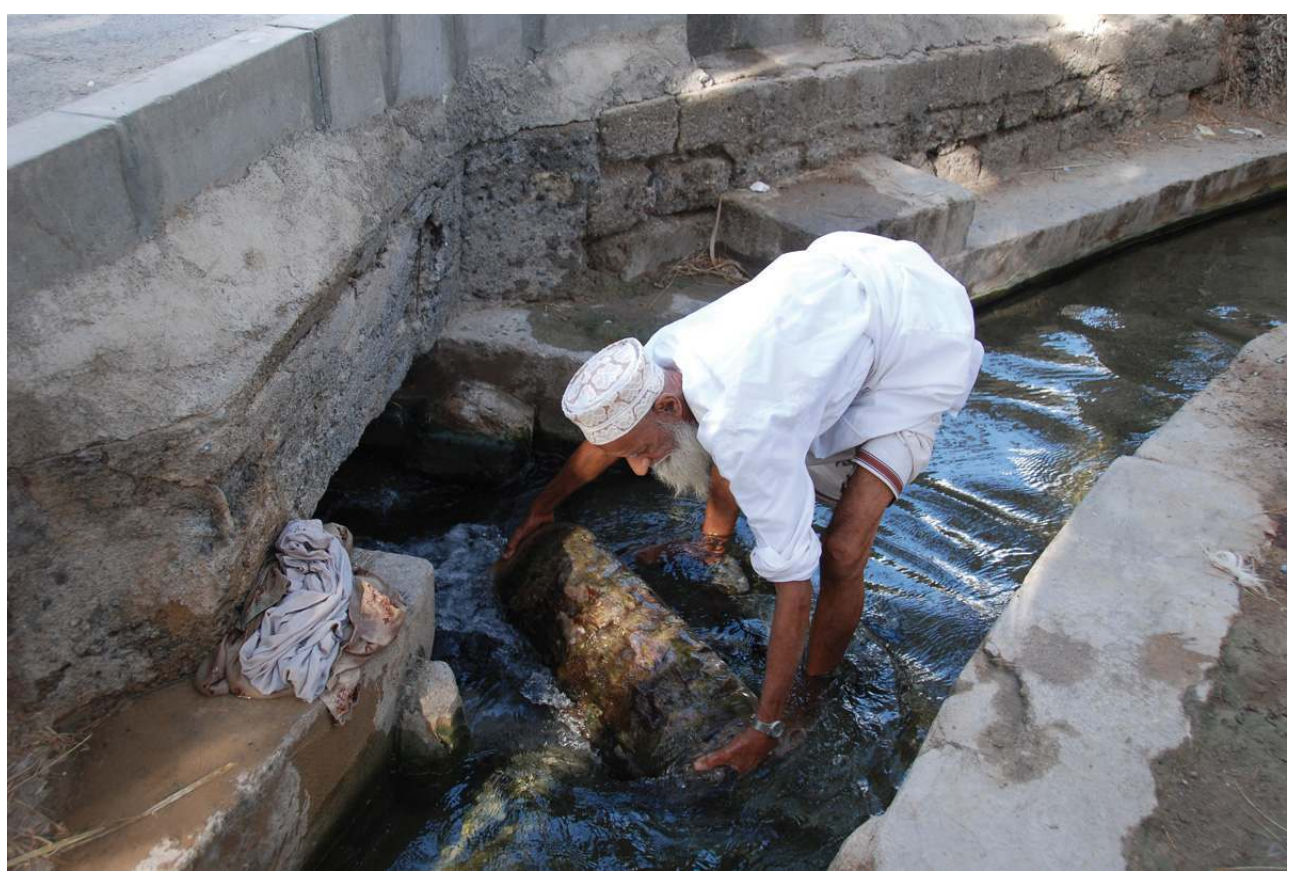

(c) Julien Charbonnier

Les oasiens utilisent les lignes, qui sont de longueur variable et plus ou moins éloignées $\mathrm{du}$ style, à tour de rôle, afin de compenser l'allongement, puis le raccourcissement de l'ombre de ce dernier en fonction des saisons. La plus longue, au nord, est utilisée en hiver et la plus courte, au sud, au cours de l'été.

Les autres cadrans d'Adam fonctionnent suivant un principe proche. Celui du falaj al-'Ayn, localisé à l'est de l'oasis, est quasiment identique, mais il dispose de seulement six lignes parallèles. Sur le cadran du falaj al-Filaij, les plots métalliques sont remplacés par des galets (hașâ) (Figure 8). La khashaba du falaj al-Shâr'a est d'un modèle un peu différent. En fonction des saisons, les baguettes de bois sont disposées à divers endroits de lignes orientées nord-sud (Figures 9 et 10). 
Figure 8. Le cadran solaire du falaj al-Filaij, vu depuis le sud-ouest

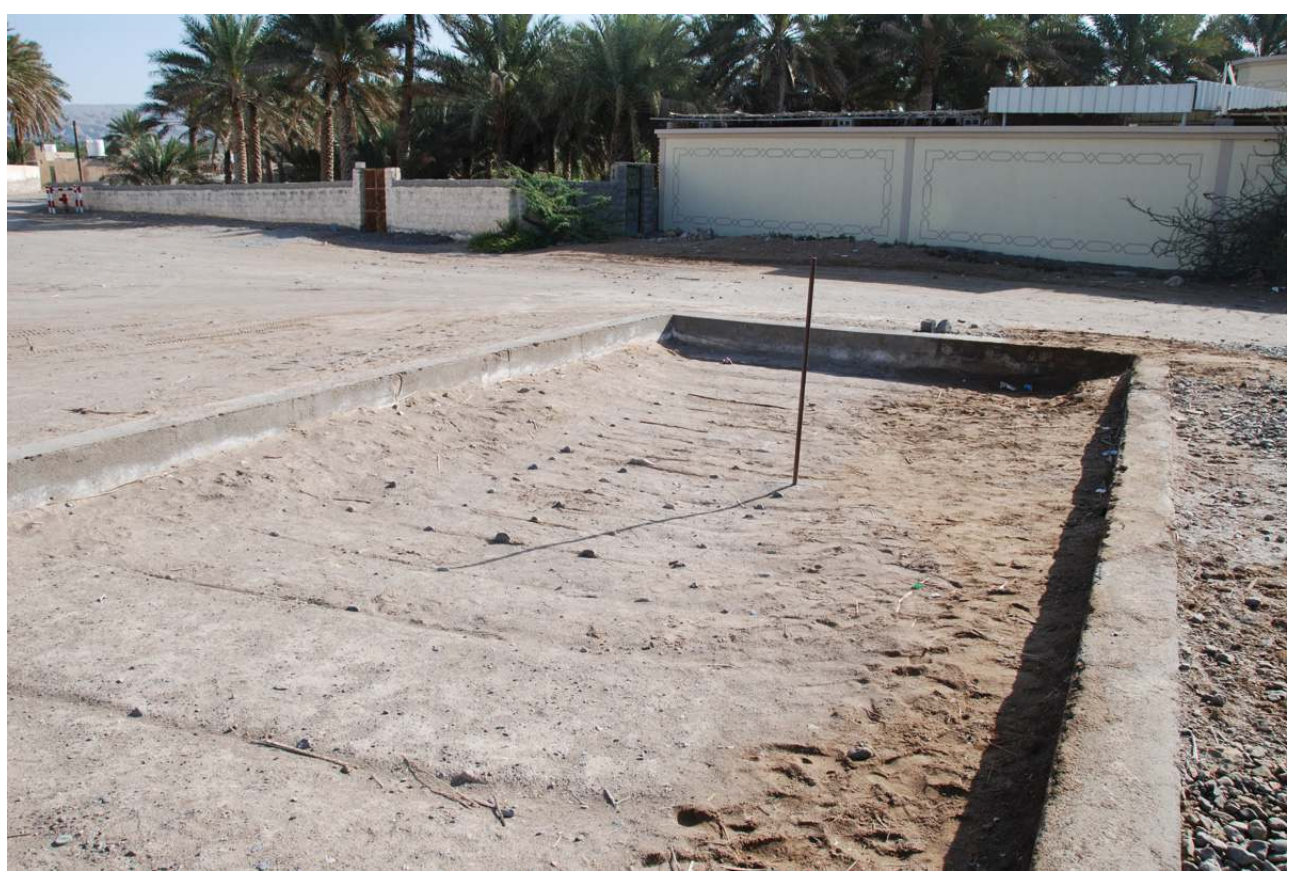

(c) Julien Charbonnier

Figure 9. Le cadran solaire du falaj al-Shâr'a, vu depuis le sud

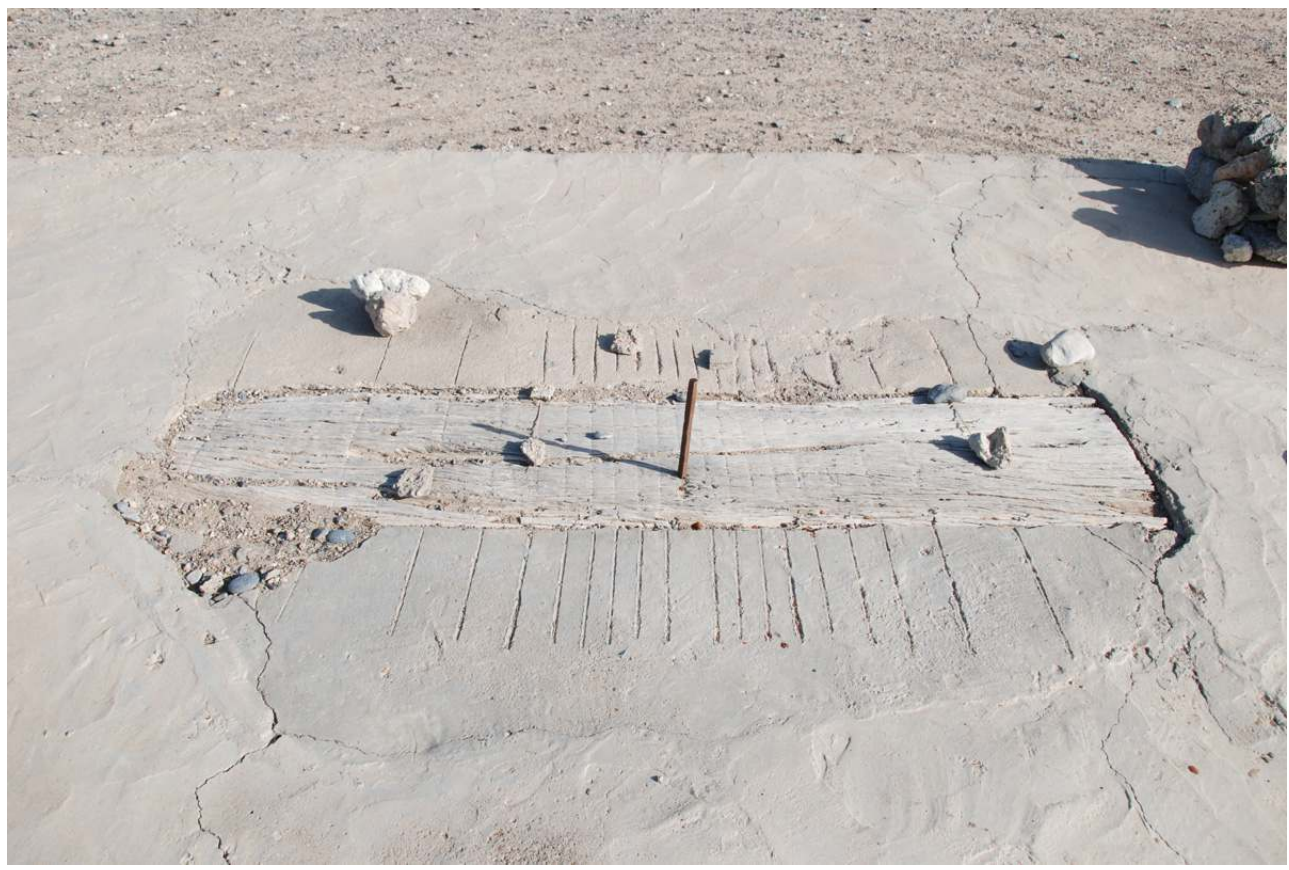

(c) Julien Charbonnier 

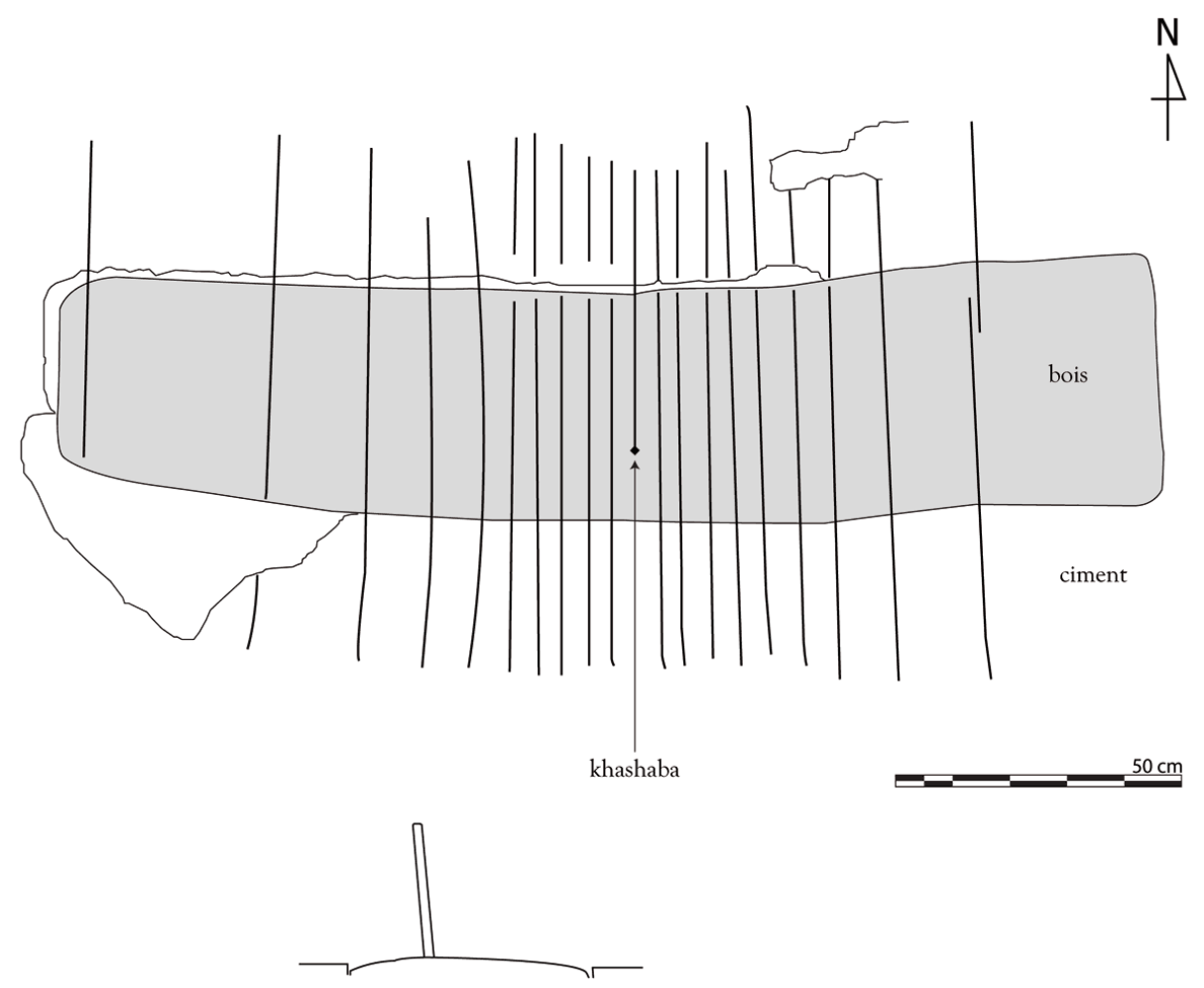

Relevé Julien Charbonnier

Des outils de mesure du temps similaires ont été employés dans tout le nord de l'Oman. On en connaît par exemple dans le wâdî Ghûl ou dans le village de Qarya (Nash $2011: 23$ et 34), tous deux situés dans la région d'al-Ḥamrâ, au nord de la ville de Bahlâ'.

\section{L'inscription spatiale des tours d'eau}

D'une part à l'autre, l'eau peut être envoyée à des points opposés du système d'irrigation. Son déplacement a été enregistré pendant deux jours afin de le démontrer.

Dans la journée du 14 février 2011, qui correspond au badda al-Khâldiya, les vingt-quatre athar ont été répartis entre onze personnes. Les voici citées dans l'ordre :

- Ahmad bin Saïd (6 athar)

- Mohammad bin Saïd (1 athar et demi)

- Mohammad bin Amir (2 athar et demi)

- Kamsa bin Ali (1 athar)

- Yacoub (1 athar)

- Saïd et Ahmad bin Suleyman (2 athar et demi)

- Abdallah bin Saïd (1 athar et demi)

- Sharif bin Khalfan (2 athar)

- Moubarak bin Sad (3 athar)

- Mohammad bin Hamoud (1 athar)

- Ahmad bin Salem (2 athar) 
La figure 11 représente la position des baguettes sur le cadran au cours de la journée du 14 février 2011. La figure 12 montre quant à elle que les parts d'eau n'ont pas été réparties en prenant en compte un quelconque ordre spatial.

Figure 11. Répartition des baguettes sur le cadran du falaj al-Mâleh dans la journée du 14 février 2011

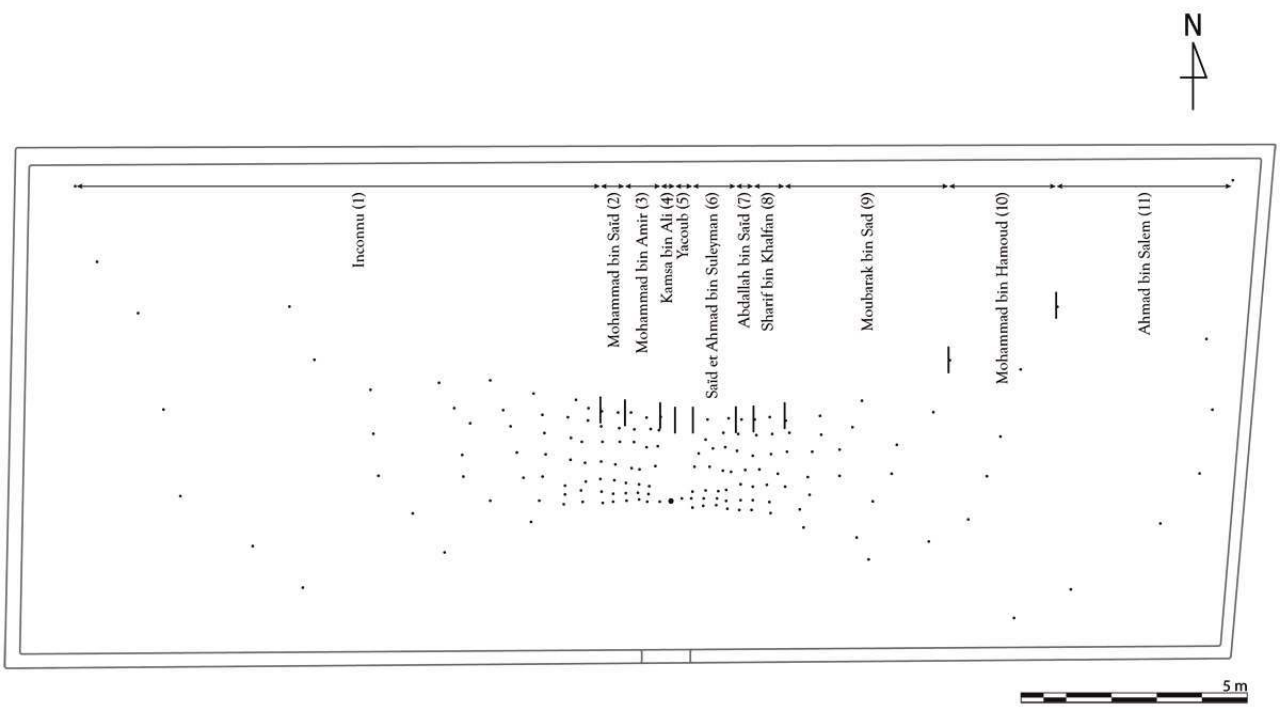

Infographie Julien Charbonnier

Figure 12. Plan des canaux du falaj al-Mâleh et localisation des parcelles irriguées dans la journée du 14 février 2011, classées par ordre d'alimentation

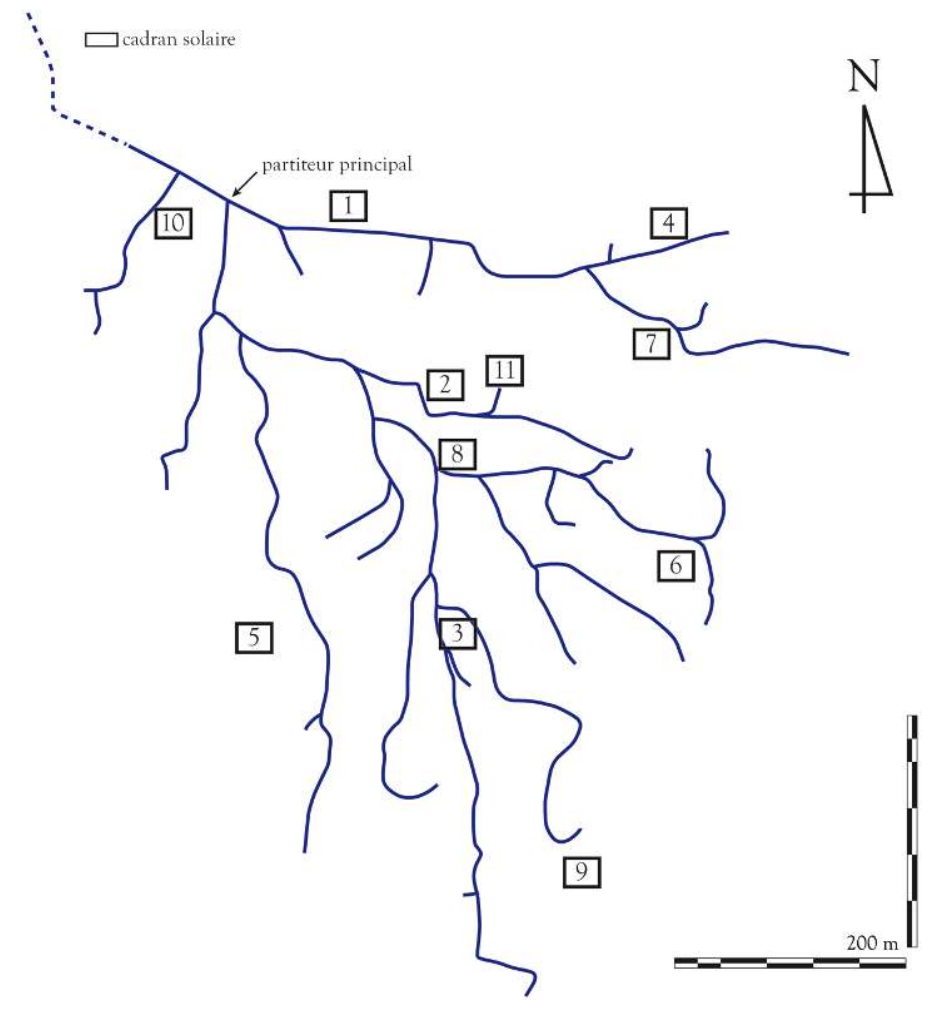

Infographie Julien Charbonnier 


\section{fut le suivant :}

- Ali bin Ahmad (1 athar)

- Houd bin Ahmad (2 athar)

- Mosquée (1 athar)

- Ahmad bin Nasser (3,5 athar)

- Moubarak bin Sad (5,5 athar)

- Mosquée (1 athar)

- Mohammad bin Abdallah (1/2 athar)

- Mohammad bin Talib (1,5 athar)

- Saïd bin Nasser (3 athar)

- Mosquée (2 athar)

- Saïd Eïd (1,5 athar)

- irrigant qui n'est pas venu prendre son tour (1 athar)

- Yacoub (1/2 athar).

La figure 13 montre la répartition spatiale de l'eau dans la journée du 22 février 2011.

On remarquera qu'un des irrigants n'est pas venu récupérer son tour d'eau. Les terres appartenant à la mosquée ont été irriguées par trois fois. L'une de ces parcelles était située sur le système d'irrigation du falaj al-'Ayn. L'eau a pu passer d'un réseau de canaux à l'autre grâce à un déversoir.

C'est une logique purement temporelle qui préside à la distribution de l'eau. Chaque irrigant vient récupérer ses parts, qui sont réparties de manière aléatoire le long du cycle, à heure fixe et peut alors envoyer l'eau sur la parcelle de son choix. D'un cycle à l'autre, chaque badda est donc également légèrement différent en termes de répartition spatiale de l'eau. 
Figure 13. Plan des canaux du falaj al-Mâleh et localisation des parcelles irriguées dans la journée du 22 février 2011 , classées par ordre d'alimentation

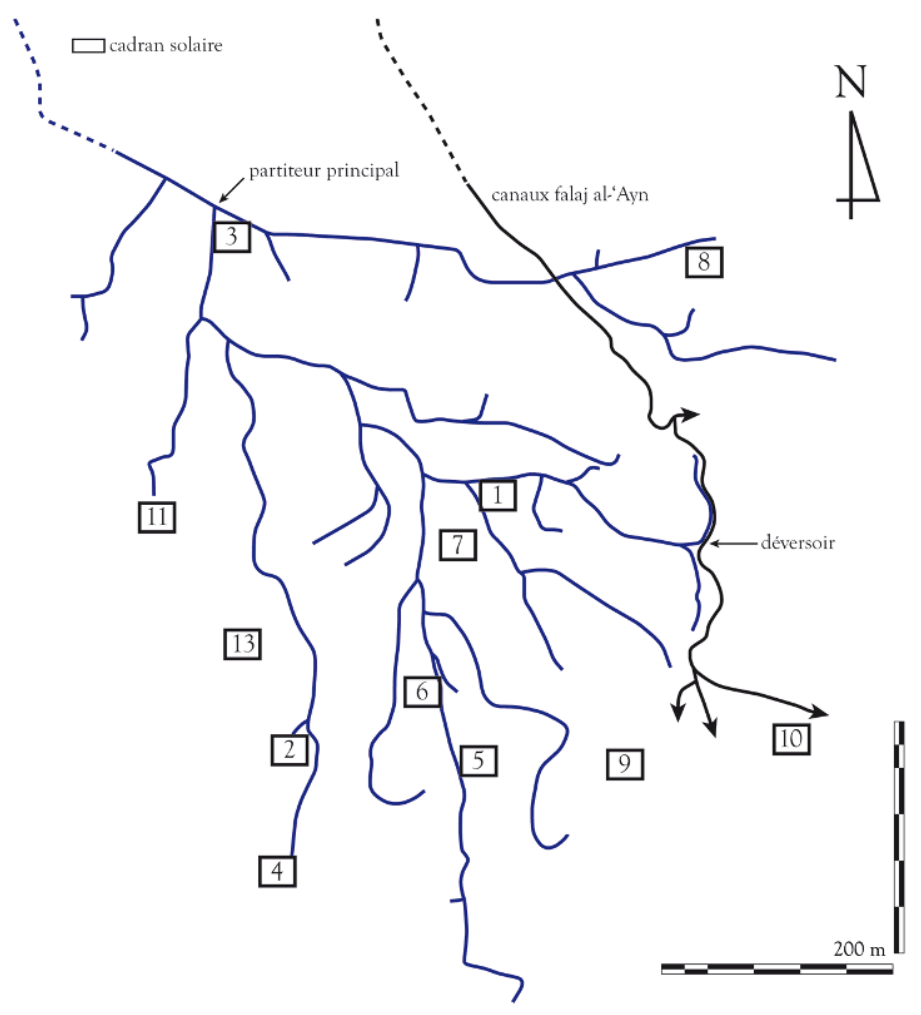

Infographie Julien Charbonnier

Ce mode de gestion, temporel, caractérise tous les aflâj d'Adam, et d'Oman plus généralement. Il contraste nettement avec celui en vigueur au Yémen, à l'autre extrémité de la Péninsule Arabique. Dans cette région, on utilise surtout l'eau de crue des wâdî, qui est détournée à l'aide de digues déflectrices. Au sein de chaque système, il est de règle que les parcelles soient alimentées les unes à la suite des autres, d'amont en aval (Pelat 2005 : 249 ; Bédoucha $2004: 287$ ). Lorsque l'eau a atteint la hauteur voulue dans un champ, on l'envoie dans celui situé en contrebas (Bédoucha 2004 : 295). Ceux implantés le plus aval ne sont alors irrigués qu'occasionnellement (Bonnenfant 1997 : 18-20).

Dans les oasis omanaises alimentées par des aflâj, l'eau doit parfois parcourir une distance importante pour passer d'un champ à l'autre. C'est l'une des contraintes posées par la gestion temporelle de la ressource. L'eau circule en effet lentement dans le réseau de canaux d'al-Mâleh ( $2 \mathrm{~km} / \mathrm{h}$ en moyenne), elle peut mettre plus de 20 minutes pour rejoindre une parcelle depuis le partiteur le plus en amont. Les irrigants doivent prendre en compte ce temps de déplacement, car il les met à inégalité pendant les changements de tour. En effet, s'ils déplacent les vannes au moment exact où l'extrémité de l'ombre touche la baguette, la quantité d'eau reçue par le champ en cours d'alimentation sera différente si la parcelle suivante est alimentée par le même ou par un autre canal secondaire.

Imaginons que le champ qui suit est desservi par un autre canal secondaire. L'eau est alors déviée depuis le partiteur le plus en amont. Au moment où l'irrigant change la vanne, il reste un volume d'eau important tout au long du premier canal secondaire, volume qui bénéficie à la première parcelle (Figure 14A). Dans le cas où le champ suivant 
est desservi par le même canal secondaire, le partiteur actionné est bien plus proche de la précédente parcelle. Si la vanne est déplacée au moment exact où l'ombre touche la baguette, ou peu après si l'on prend en compte le temps de déplacement de l'irrigant, le volume d'eau qui finira de transiter vers la parcelle précédente sera moins important que dans le cas précédent (Figure 14B), voire même nul si le deuxième champ est situé plus en aval (Figure 14C).

Figure 14. Parcours de l'eau dans la palmeraie
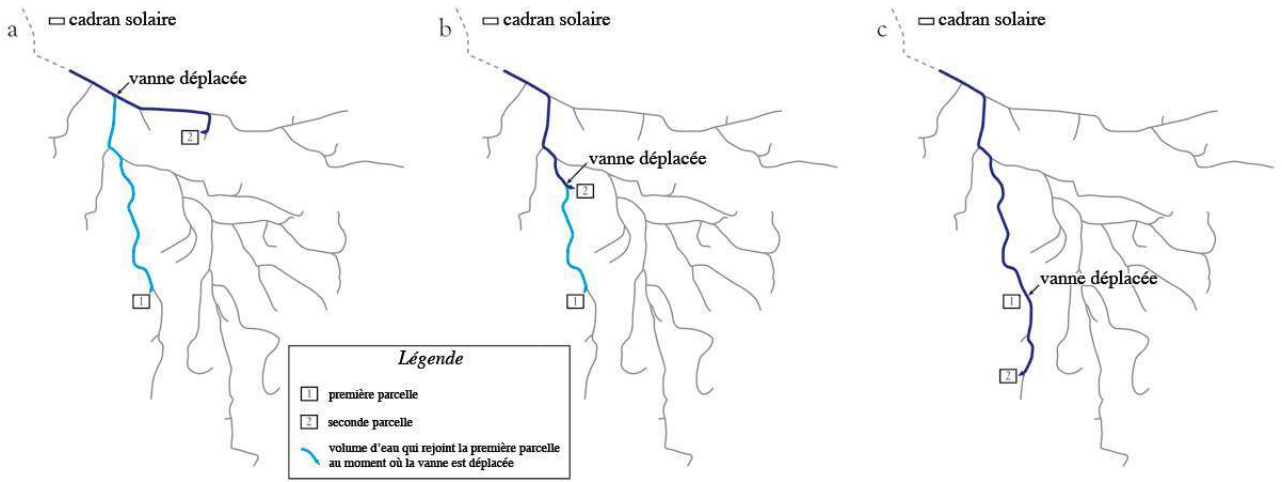

A : si la parcelle suivante est alimentée par un autre canal secondaire ; B : si la parcelle suivante est située sur le même canal primaire en amont ; C : si la parcelle suivante est située sur le même canal primaire en aval

Infographie Julien Charbonnier

Pour compenser cette inégalité, l'oasien dont c'est le tour d'irriguer sa parcelle, et qui se trouve dans le deuxième cas de figure, se place au niveau du partiteur le plus en amont qui dessert les canaux secondaires - et jette dans le falaj quelques miettes de feuille sèche. Il attend ensuite qu'elles atteignent le partiteur à manipuler, et donc qu'un certain volume d'eau ait eu le temps de rejoindre le champ précédent, pour déplacer les vannes.

\section{Les avantages d'une répartition temporelle de l'eau}

Les aflâj permettant d'obtenir de l'eau tout au long de l'année, les hommes peuvent se libérer des contraintes climatiques. Les possibilités de culture ne dépendent plus du rythme des précipitations. Comme nous l'avons souligné, ces ouvrages imposent cependant aux hommes un nouveau rythme de vie et de travail, marqué par le cycle de rotation de l'eau. Les utilisateurs d'un falaj ne peuvent alimenter leurs terres à leur guise. Ainsi, le qanât " se transforme le plus souvent, à long terme, en un système vulnérable et exigeant, générateur de rythmes humains contraignants pour ses utilisateurs » (Lombard 1991 : 84).

Se plier au rythme du cycle de l'eau peut poser problème, notamment en ce qui concerne les cultures maraîchères. Celles-ci nécessitent en général un apport d'eau très fréquent, voire journalier. Si les parts d'un irrigant sont très éloignées dans le temps, cela limitera ses possibilités de culture.

Par ailleurs, la répartition purement temporelle des parts d'eau implique une gestion complexe de la ressource, comme nous l'avons vu. Il faut la partager en permanence, de jour comme de nuit, manipuler toute une série de vannes pour l'envoyer à l'endroit voulu, utiliser des outils élaborés de division du temps et prendre en compte son temps 
de déplacement. Le fait que l'eau puisse, d'un tour à l'autre, transiter d'une extrémité à l'autre de la palmeraie augmente également les pertes par infiltration et évaporation.

Cependant, une répartition spatiale fixe de l'eau serait encore plus contraignante pour les utilisateurs d'un falaj, car le système serait extrêmement rigide. Un irrigant ne pouvant pas choisir laquelle de ses parcelles il souhaite alimenter, il serait obligé d'envoyer l'eau vers un champ même quand celui-ci n'en a pas besoin et qu'aucune culture ne se trouve dessus. Inversement, il devrait parfois attendre 14 jours, un cycle complet, pour irriguer certaines parcelles.

Une gestion purement temporelle est par certains aspects contraignante, mais donne une certaine souplesse au système. Les irrigants peuvent intervertir leurs parts. Ils ont également la possibilité d'acheter des athar au cours de ventes aux enchères qui ont lieu toutes les deux semaines. Ils les récupèrent au cours du badda al-Q'âida. L'argent récolté permet de financer une partie des opérations d'entretien du falaj. Les tours d'eau du badda al-Zayda sont quant à eux vendus à l'année. Le partage temporel de l'eau met en outre les utilisateurs à égalité devant les variations annuelles de débit des aflâj (liées aux oscillations de la nappe).

\section{Falaj et phœniciculture}

Le falaj est par ailleurs bien adapté à la phœniciculture. Les palmiers dattiers (Phoenix dactylifera L.), comme tous les arbres fruitiers, ont besoin d'être arrosés tout au long de l'année. L'irrigation à partir des eaux de crue, qui est encore pratiquée de nos jours dans les montagnes, pose problème dans la mesure où les crues ne se produisent essentiellement qu'au cours de l'hiver. La disponibilité épisodique de l'eau d'un falaj, liée au mode de partage en vigueur en Oman, constitue un autre avantage de cette technique d'irrigation. Les palmiers doivent en effet être irrigués ponctuellement. Au Maroc, il a été évalué qu'il est nécessaire de les alimenter tous les 3 à 6 jours en été et tous les 10 à 15 jours en hiver (Peyron 2000: 38). L'aspect cyclique de la distribution de l'eau convient ainsi parfaitement au palmier dattier ${ }^{7}$. On peut ainsi se demander si l'adoption ou le développement du falaj n'a pas favorisé sa culture, et vice-versa.

Le palmier est un arbre fondamental de l'agriculture d'Arabie du sud-est et ce depuis au moins le III ${ }^{\mathrm{e}}$ millénaire avant J.-C. De nombreux vestiges de dattes ont en effet été mis au jour sur certains sites datés de l'âge du Bronze ancien (Costantini et Cleuziou 1980; Tengberg 2003). L'importance du palmier s'explique de plusieurs façons. La datte, tout d'abord, a un apport calorique inégalé pour un fruit - environ 300 calories pour $100 \mathrm{~g}$ (Peyron $2000: 22$ ) - et, séchée, se conserve pendant plusieurs mois. Les arbres protègent quant à eux les autres cultures de la chaleur, du soleil et du vent (Tengberg 2003 : 232-233). Au sein des palmeraies, la température de l'air est plus faible qu'en dehors, limitant l'évaporation.

Les plus anciens aflâj reconnus en Arabie du sud-est alimentaient des établissements datant de l'âge du Fer II (1100-600 avant J.-C. ; al-Tikriti 2002 et 2010 ; Benoist et al. 2012 ; Charbonnier soumis). L'adoption de cette technique d'irrigation a peut-être favorisé le développement des palmeraies dès cette époque. Le falaj facilitant l'exploitation des nappes souterraines, de nouvelles oasis ont également pu être créées sur le piémont des montagnes (Magee 1998 : 51). 


\section{L'abandon des systèmes traditionnels de partage de l'eau}

40 L'espace apparait comme subordonné au temps dans l'oasis d'Adam. Un tel système de captage et de répartition de l'eau, si complexe à gérer, nécessite une coopération constante entre irrigants et une cohésion forte au sein des communautés. On peut penser que ces sociétés se sont façonnées autour du partage de l'eau depuis l'apparition du falaj en Arabie du sud-est, au cours de l'âge du Fer, il y a environ 3000 ans (al-Tikriti 2002). La gestion de l'eau est au centre de la vie sociale dans les oasis, car la survie du groupe en dépend et les rapports tissés autour de sa répartition sont un élément structurant.

41 Par son organisation, l'oasis d'Adam témoigne de l'existence de communautés soudées: au sein de la palmeraie, les habitants vivaient autrefois dans quelques villages compacts ${ }^{8}$ et non pas dans des maisons dispersées. Les quelques bâtiments indépendants disséminés entre les parcelles étaient réservés aux saisonniers venus récolter les dattes ou labourer les champs.

42 Le cadran était le lieu autour duquel se rassemblaient et venaient discuter les hommes de l'oasis. Ils pouvaient débattre des questions liées au falaj et surveiller le bon déroulement du cycle, les contestations n'étant pas rares au sujet du partage de la ressource. La racine du mot muhâara peut d'ailleurs signifier " être témoin de " (Nash 2009: 54) ${ }^{9}$. C'est pour cette raison que cette technique est encore utilisée par quelques hommes âgés de l'oasis : le cadran est un lieu d'échange et il permet à tous de vérifier que personne n'est floué. Ainsi, il n'est pas rare qu'un premier irrigant pose une baguette, puis qu'un second vienne la déplacer légèrement, car il en conteste la position. Quelques millimètres de différence sur le cadran représentent en effet plusieurs mètres cubes d'eau. N'oublions pas qu'il s'agit d'une ressource rare dans cette région aride d'Oman. Ces négociations ne pourraient pas avoir lieu s'ils avaient recours aux montres. Le calcul des athar à l'aide des étoiles n'est plus pratiqué à Adam, car la luminosité émise par les habitations et les aménagements urbains - Adam est une bourgade en pleine expansion - ne permet plus la lecture correcte du mouvement des astres au cours de la nuit.

Les techniques traditionnelles de répartition de l'eau se perdent, car les communautés d'oasis se désagrègent peu à peu. Seule une poignée de personnes âgées savent encore utiliser le cadran solaire. Elles sont en général rémunérées par les autres irrigants pour s'occuper de leurs terres. Les jeunes générations font des études, puis partent travailler en ville. Elles conservent souvent des parcelles dans l'oasis, mais n'y vivent plus qu'épisodiquement. Les liens communautaires se distendent et les personnes n'éprouvent plus le besoin de bâtir des relations sociales autour de la distribution de l'eau. Les jardins individuels, séparés les uns des autres, se multiplient en aval de l'oasis. Bien qu'ils reflètent un attachement à la possession d'un lopin de terre, ils sont bien souvent cultivés par des ouvriers indiens ou bengalis et sont alimentés par des puits motorisés. Ces derniers permettent d'obtenir de l'eau sans limitation et affranchissent les propriétaires des contraintes du partage et de la gestion de la ressource en commun. L'eau n'est plus alors un trait d'union. 


\section{Conclusion} documenter. Ins devront être comparés à celui en vigueur sur le falaj al-Male. De manière générale, trop peu de chercheurs se sont intéressés aux méthodes de gestion de l'eau en Oman et à leur impact sur la société. Une lacune qui devra être comblée avant que les communautés gérant les oasis ne disparaissent. Les aflâj ne correspondent pas seulement à des ouvrages hydrauliques, mais renvoient aussi à des pratiques sociales et à une tradition essentiellement orale.

Nous tenons ici à remercier les membres de la Mission archéologique française à Adam, et tout particulièrement ses directeurs successifs, Jessica Giraud et Guillaume Gernez, ainsi qu'Ali Hamood al-Mahrooqi, du Ministère de la Culture et du Patrimoine, pour leur appui tout au long des trois missions de terrain qui ont permis la rédaction du présent article.

Nous adressons également nos remerciements à Margareta Tengberg qui, au travers du programme ANR Phønix, a encouragé cette étude. Celle-ci n'aurait évidemment pu aboutir sans l'aide précieuse des irrigants d'Adam, qui ont fait montre d'une extrême patience pour nous expliquer le fonctionnement du cadran. Toute notre gratitude leur revient.

\section{BIBLIOGRAPHIE}

al-Ghafri A., Inoue T. \& Nagasawa T. 2004 - Irrigation Scheduling of Aflaj of Oman : Methods and Modernization, Université d'Hokkaïdo, www.inweh.unu.edu/drylands/docs/Publications/ AlGhafri.pdf.

al-Tikriti W.Y. 2002 - The south-east Arabian origin of the falaj system. Proceedings of the Seminar for Arabian Studies 32 : 117-138.

al-Tikriti W.Y. 2010 - Heading North : an ancient caravan route and the impact of the falaj system on the Iron Age culture. In Avanzini A. (Ed.), Eastern Arabia in the First Millennium BC. Rome, «L'Erma » di Bretschneider, : 227-247. (Arabia Antiqua ; 6).

Bédoucha G. 2004 - Éclipse de lune au Yémen. Émotions et désarrois d'une ethnologue. Paris, Odile Jacob.

Benoist A., Bernard V., Charbonnier J., Goy J., Hamel A. \& Sagory T. 2012 - Une occupation de l'Âge du Fer à Masafi : travaux récents de la Mission Archéologique Française aux E.A.U. dans l'Émirat de Fujairah. Chroniques Yéménites, 17 [en ligne]. http://cy.revues.org/1803 
Bonnenfant P. 1997 - La maîtrise de l'eau dans le wadi Zabid, Yémen. Bulletin de l'association des géographes français 74/1, « Espaces du monde arabe » : 12-24.

Charbonnier J. soumis - Spatial organization of pre-Islamic oases in Southeast Arabia: the influence of the falaj, Water History.

Cleuziou S. \& Costantini L. 1980 - Premiers éléments sur l'agriculture protohistorique de l'Arabie orientale. Paléorient $6: 245-251$.

Dutton R.W. 1989 - Aflaj renewal in Araqi : a village case study from Oman. In Beaumont P., Bonine M. \& McLachlan K. (Ed.), Qanat, Kariz et Khattara : Traditionnal Water Systems in the Middle East and North Africa. Londres, The Middle East Centre, School of Oriental and African Studies, University of London, et Cambridgeshire, Middle East and North African Studies Press : 237-256.

Lombard P. 1991 - Du rythme naturel au rythme humain : vie et mort d'une technique traditionnelle, le qanāt. In Cauvin M.-C. (Dir.), Rites et rythmes agraires. Lyon, Maison de l'Orient ; Paris, Diffusion De Boccard : 69-87. (Travaux de la Maison de l'Orient ; 20).

Magee P. 1998 - Settlement patterns, polities and regional complexity in the Southeast Arabian Iron Age. Paléorient 24/2 : 49-60.

Nash H. 2009 - Water Management: the Use of Stars in Oman. Thèse de doctorat, Université d'Exeter.

Nash H. 2011 - Water Management: the Use of Stars in Oman. Oxford, Archaeopress. (Society for Arabian Studies Monographs ; 11).

Pelat F. 2005 - L'intrusion de l'état dans les droits d'eau au Yémen : le temps des coutumes seraitil révolu ?. In Dupret B. \& Burgat F. (Dir.), Le Shaykh et le Procureur. Systèmes coutumiers et pratiques juridiques au Yémen et en Égypte. Le Caire, Centre d'études et de documentation économiques, juridiques et sociales (CEDEJ) : 247-273.

Peyron G. 2000 - Cultiver le palmier-dattier. Montpellier, Cirad.

Tengberg M. 2003 - Archaeobotany in the Oman peninsula and the role of Eastern Arabia in the spread of African crops. In Neumann K., Butler A. \& Kahlheber S. (Ed.), Food, Fuel and Fields :

Progress in African Archaeobotany. Cologne, Heinrich Barth Institut : 229-237. (Africa Praehistorica ; 15).

Trousset P. 1986 - Les oasis présahariennes dans l'Antiquité : partage de l'eau et division du temps. Antiquités Africaines $22: 163$.

Wilkinson J.C. 1977 - Water and tribal settlement in South-East Arabia, a study of the Afläj of Oman. Oxford, Clarendon Press.

\section{NOTES}

1. Cette répartition est le produit d'une histoire. Elle a été façonnée par les héritages, les ventes et les échanges.

2. En réalité, l'athar est de longueur variable suivant les oasis.

3. L'ordre de distribution de l'eau peut être partiellement altéré, par exemple si deux irrigants échangent ou se prêtent des parts d'eau. Celles-ci peuvent également être le bien commun des membres d'une même famille et donc profiter à des personnes différentes d'un tour à l'autre. Les parts sont en outre parfois vendues.

4. Khashab désigne le bois en arabe. Le style était appelé khashaba car il était autrefois en bois.

5. Le lieu où l'on observe les étoiles est aussi appelé muhâạar (Nash 2009 : 54). 
6. Les vannes (jâla) sont taillées dans des troncs de palmier. Elles sont calfatées à l'aide de morceaux de tissus et calées avec des pierres.

7. Dans les montagnes d'al-Ḥajar, on exploite principalement les sources pour l'alimentation des palmeraies, mais leur gestion est identique à celle du falaj dầûdî, car là encore l'eau coule en permanence.

8. Au cours des vingt dernières années, ils ont été relogés dans des quartiers modernes édifiés à la périphérie de la palmeraie.

9. La définition la plus courante de muhâdara est « exposé oral », « conférence » ou « cours ».

\section{RÉSUMÉS}

Parce qu'elle délivre de l'eau en permanence, la technique d'irrigation utilisée sur le piémont des montagnes d'Oman, le falaj, impose à ses utilisateurs un partage temporel de la ressource. Chaque irrigant possède un nombre variable de parts d'eau de 30 minutes. En l'absence de montre, les utilisateurs de falaj ont utilisé des cadrans solaires pour calculer ces parts d'eau. Cet outil est encore employé dans de nombreuses oasis d'Oman, dont celle d'Adam, qui est gérée de manière traditionnelle. Au sein de sa palmeraie, l'eau n'est pas répartie de façon spatiale. Chaque irrigant peut, au moment où il la récupère, utiliser sa part sur la parcelle de son choix. Ce mode de répartition contraste avec celui en vigueur en Arabie du sud-ouest, où l'eau alimente les champs les uns à la suite des autres, d'amont en aval. La gestion temporelle des parts pose des contraintes techniques - l'eau doit parfois se déplacer sur une grande distance pour passer d'une parcelle à la suivante -, mais a pour avantage d'offrir une plus grande souplesse dans la culture des plantes annuelles. Elle apparaît également très bien adaptée au palmier dattier, un arbre fondamental pour l'écologie des oasis.

The irrigation technique prevailing in Oman oases, the falaj, delivers water year round. The users of a falaj have to divide its water on a temporal basis. Each of them is allocated a certain number of 30-minute water shares. Sundials were traditionally used to calculate these shares. This is still the case in the oasis of Adam. The inhabitants of this oasis do not take into account a spatial order to allocate water. Each of them can use his share for any one of the plots he owns. This method of distribution contrasts with the one used in Southwestern Arabia. In that region, the water goes through the entire irrigation system from upstream to downstream, plot after plot. The temporal management of water used in Oman presents some technical inconvenience. Between two plots, water must sometimes run long distance. However, this method allows the system to be more flexible and is favourable to the cultivation of annual plants. It is also suitable for the date palm, the key plant of the oasis.

\section{INDEX}

Keywords : date palm, Oman, sundial

Mots-clés : cadran solaire, falaj, irrigation, oasis, palmier dattier

Index géographique : Âdam, Arabie 


\section{AUTEUR}

\section{JULIEN CHARBONNIER}

UMR 7041 Archéologies et Sciences de l'Antiquité

Maison René Ginouvès

21, allée de l'Université

F-92023 Nanterre Cedex

julcharbonnier@gmail.com 